\title{
Adsorption of starch, amylose, amylopectin and glucose monomer and their effect on the flotation of hematite and quartz
}

\author{
S. Pavlovic ${ }^{a}$, P.R.G. Brandao ${ }^{\text {b,* }}$ \\ ${ }^{a}$ Federal University of Ouro Preto, Ouro Preto, 35400-000, Brazil \\ ${ }^{\mathrm{b}}$ Department of Mining Engineering, Federal University of Minas Gerais, Rua Espirito Santo, 35-s/702, Belo Horizonte, MG 30160-030, Brazil
}

Received 8 May 2003; accepted 24 June 2003

\begin{abstract}
The depressant effect of corn starch, its polysaccharide components (amylose and amylopectin), the monomer glucose and the dimer maltose were studied on hematite and quartz, by means of infrared spectrometry, adsorption isotherms determination and microflotation tests. All the carbohydrates tested have been effective in maintaining hematite hydrophilic, including glucose and maltose; as for quartz, only a flocculation action (mainly by amylopectin) had a mild effect in decreasing its floatability by the amine collector. FTIR studies confirmed that the carbohydrates adsorbed intensively onto hematite and the spectra of the adsorbed polymers and monomer were very similar, even though the non-adsorbed monomer spectrum was markedly different. The common adsorption mechanism indicated is a surface reaction involving the iron ion.
\end{abstract}

(C) 2003 Elsevier Ltd. All rights reserved.

Keywords: Mineral processing; Iron ores; Flotation depressants

\section{Introduction}

The method presently used in the industrial flotation of itabiritic iron ores is the reverse one, where quartz is the froth product; decyletheramine acetate is the main collector and starch is the depressor for the iron minerals. Starch and other polysaccharides are widely utilized in the mineral processing industry as depressants in froth flotation and as flocculants. Despite their wide application, the interaction mechanisms between the polysaccharides and mineral surfaces have not yet been well established. Several hypotheses regarding polysaccharide adsorption mechanism, principally hydrogen bonding, hydrophobic interactions, chemical complexation and acid/basic interactions have been proposed (Liu et al., 2000).

Starch is a complex natural non-ionic polymer, consisting of two fractions: a linear polymer amylose which is made of $\mathrm{D}$-glucose monomers joined by $\mathrm{C} 1-\mathrm{C} 4$ bonds and a branched polymer amylopectin which contains the

\footnotetext{
${ }^{*}$ Corresponding author. Tel.: +55-31-3238-1967; fax: +55-31-32381966.

E-mail address: pbrandao@demin.ufmg.br (P.R.G. Brandao).
}

same monomers joined also by $\mathrm{C} 1-\mathrm{C} 6$ linkages (Peres and Correa, 1996).

In the starch polymer only three hydroxyl groups of the cyclic glucose units are free and may rotate to one side of the molecule ring, making that side more hydrophilic. The opposite side is consequently slightly hydrophobic due to the exposed - $\mathrm{CH}$ groups. In fact in aqueous solutions amylose forms a helix with six glucose monomers per turn. The interior of the helix is hydrophobic, whereas the outer shell is hydrophilic (Liu et al., 2000).

The objective of this study was to improve the understanding of interaction of starch with the minerals hematite and quartz and to contribute to explain the selectivity in iron ore flotation.

\section{Materials and methods}

\subsection{Materials}

The samples were coarse crystalline minerals having a pure and compact appearance obtained from Minas Gerais State, Brazil. They were carefully ground to the suitable size distribution. The $\mathrm{Fe}_{2} \mathrm{O}_{3}$ content of the 
hematite sample was $99.0 \%$ and the $\mathrm{SiO}_{2}$ content of the quartz sample was $99.6 \%$.

Commercial corn starch, amylose and amylopectin were supplied by Sigma and maltose and glucose by Merck. The collector was decyletheramine acetate with $50 \%$ neutralisation degree, named Flotigan EDA-B, supplied by Clariant.

\subsection{Methods}

\subsubsection{Adsorption tests}

Freshly prepared solution of causticised starch was used in experiments. One gram sample of mineral and $100 \mathrm{ml}$ of a known concentration of starch solution was added and the flask was agitated for a specified period of time. The contents of the flasks were centrifuged or filtered through quantitative filter papers and the solid was thoroughly washed with distilled water. The temperature was maintained at $25 \pm 1{ }^{\circ} \mathrm{C}$. The value of $\mathrm{pH}$ was always $10.5 \pm 0.1$. This value was previously established as the optimum for flotation of hematite (Peres and Correa, 1996).

\subsubsection{Determination of carbohydrate adsorbed}

The starch solution was centrifuged (at $5000 \mathrm{rpm}$ ) to obtain a clear solution. A given volume of the solution was pipetted into of three similar test tubes and the residual concentration of carbohydrates on a supernatant was determined using the method described by Dubois et al. (1956).

\subsubsection{Thermogravimetry measurements}

Thermogravimetry measurements were carried out to determine the absolute amount of polysaccharides on hematite and quartz surfaces, after water washing. The following conditions were used: gas nitrogen, flow rate, $25 \mathrm{ml} / \mathrm{min}$; temperature range, $25-650{ }^{\circ} \mathrm{C}$; heating rate, $10{ }^{\circ} \mathrm{C} \mathrm{min}{ }^{-1}$.

\subsubsection{Spectroscopic measurements}

Infrared spectra were measured on a FTIR spectrometer (Perkin-Elmer 1760-X model). Samples were prepared by the standard $\mathrm{KBr}$ pellet method. Spectra were collected after 32 scans at $4 \mathrm{~cm}^{-1}$ resolution.

\subsubsection{Microflotation tests}

Flotation tests were performed with a Hallimond tube. After conditioning, the mineral sample was floated for $1 \mathrm{~min}$ at $55 \mathrm{ml} / \mathrm{min}$ nitrogen gas flow.

\subsubsection{Surface area}

Specific surface area of mineral samples was determined by the BET method.

\section{Results and discussion}

The adsorption isotherms for corn starch on hematite and quartz are shown in Fig. 1. Adsorption densities were calculated from specific surface area measurements and expressed in terms of $\mathrm{mg} / \mathrm{m}^{2}$. The BET specific surface area of hematite was $3.2 \mathrm{~m}^{2} / \mathrm{g}$ and of quartz was $1.0 \mathrm{~m}^{2} / \mathrm{g}$.

The results showed that starch, amylose and amylopectin adsorption on hematite were similar. On quartz, the results were different: amylose was more abstracted that starch and amylopectin was not observed to have been abstracted. Schulz and Cooke (1953) observed the greater adsorbability of amylose over amylopectin and starch onto mineral surfaces, too. However significant errors may be have been associated with the measurements of adsorption densities, due mainly to amylose retrogradation, on centrifugation, and due to the dilution necessary in higher concentrations, in spite that blank tests were also conducted.

In order to confirm these results adsorption experiments using thermogravimetry were carried out, to determine the absolute amount of polysaccharides onto hematite and quartz surfaces, after water washing. The mass loss over the $250-375{ }^{\circ} \mathrm{C}$ range was attributed to the pyrolysis of the adsorbed polysaccharide, resulting in the liberation of carbon dioxide and water vapour. Results are shown in Fig. 2. Amylose was more adsorbed onto the two minerals. Amylose is a linear, flexible polymer displaying random coil behaviour in aqueous alkaline solution; thus, when adsorbed it may be coiled, leading to higher adsorption densities.

\subsection{Microflotation tests}

The effect of polysaccharide concentration on the floatability of hematite and quartz is shown in Fig. 3. As expected, starch was a more effective depressant for hematite than quartz. The results showed that starch,

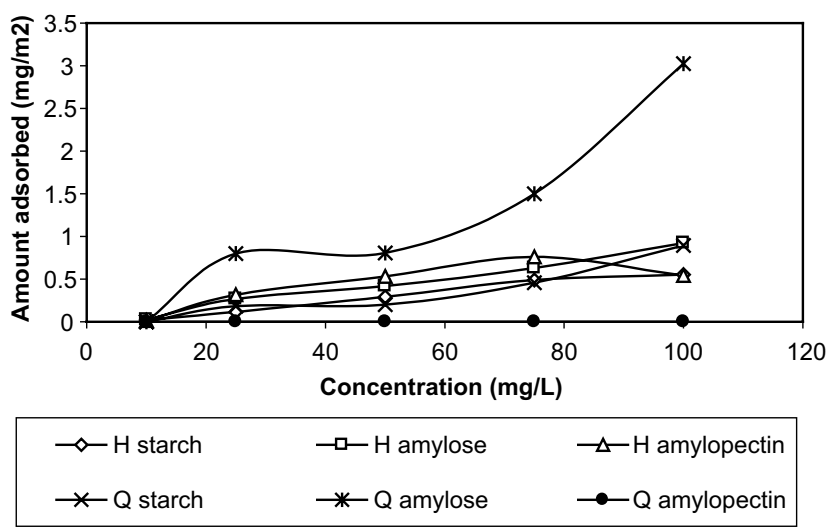

Fig. 1. Adsorption of corn starch, amylose, amylopectin onto hematite $(\mathrm{H})$ and quartz $(\mathrm{Q})$. 


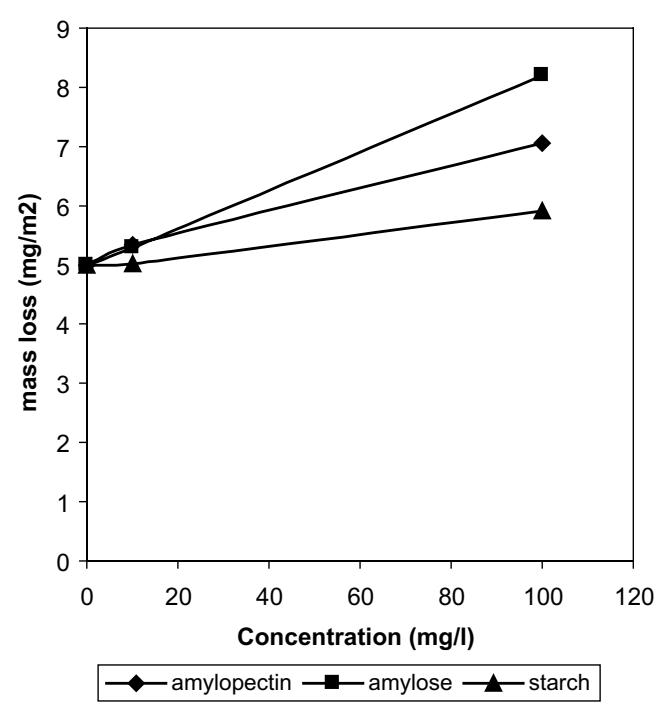

Fig. 2. Quartz conditioned with carbohydrates: mass loss measured by thermogravimetry $\left(250-375{ }^{\circ} \mathrm{C}\right.$ range).

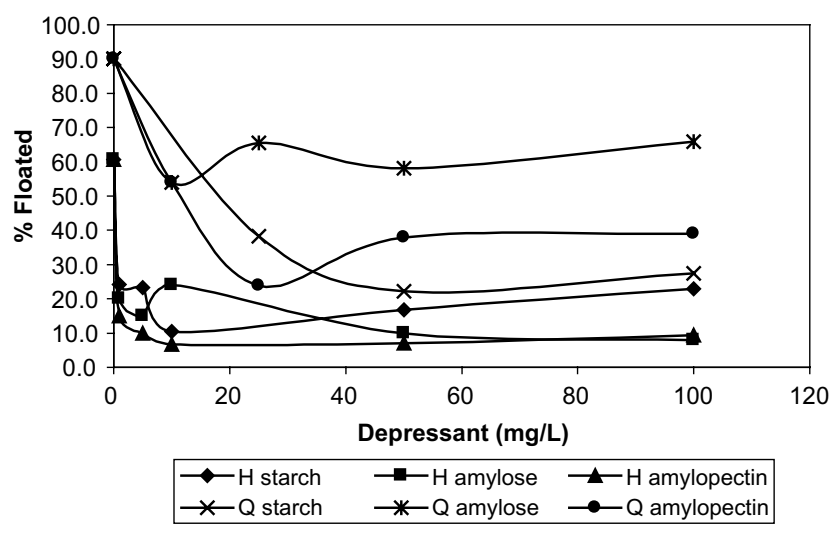

Fig. 3. Floatability of hematite and quartz as a function of starch, amylose and amylopectin depressants.

amylose and amylopectin were depressants for hematite in a very similar way. But the depression was different for quartz. Amylose showed the worst performance as quartz depressant. This results may be attributed to the fact that amylose is not a flocculant. It was also unable to flocculate hematite (Weissenborn et al., 1996). Even though the adsorption of amylopectin was extremely small, it was still efficient as a depressant for quartz. Probably, few amylopectin molecules have been able to anchor onto the surface and therefore to flocculate the quartz particles. Hogg (1999) considered the relationship between adsorption and flocculation as not clear; adsorption being only one step in such a complex phenomenon. By comparing the adsorption densities of starch, amylose and amylopectin onto hematite and quartz with their depressant action, it was not possible to correlate the depressant action to the amount of polysaccharide adsorbed, mainly for quartz.
Depressant action generally is accepted to involve the coating of a natural low energy hydrophobic surface with a hydrophilic film to prevent the attachment of air bubbles. However, there is no easy way to quantifying these two effects, flocculation and flotation.

Not withstanding this difficult, one way in which the proposition might be tested is by using monomers and dimers as depressants to flotation. If only surface properties were changed by depressants, monomers would have the same effect as polymers. Fig. 4 shows the effects on the floatability of hematite and quartz. Glucose and maltose in high concentration were depressants for hematite, but had no effect on quartz. The high concentration was necessary because glucose and maltose are highly soluble in water and only few molecules could interact with the mineral surface. These results indicate that the flocculation action is more important to the starch depressant action for quartz than surface modification.

The chemical interaction of starch, amylose, amylopectin, maltose and glucose at the mineral-water interface was established by FTIR spectroscopic analysis. Firstly, the spectra of causticised polysaccharides, at $\mathrm{pH}$ 10.5 after drying, were obtained. The spectra of carbohydrates are shown in Fig. 5. Glucose and maltose were used without dissolution. In all the spectra showed, the ordinate unit is transmittance $\%$, but is not displayed, since the data are fully comparative. The spectra of polysaccharides are similar, but band intensities for amylose were always lower, probably due to its inner molecular interactions. Spectra of glucose and maltose exhibit more absorption bands than the polymer spectra, what should be expected from simpler, smaller molecules, which have more degrees of freedom.

To simplify the interpretation, the spectra can be divided in regions. The bands in regions between 3500 $3300 \mathrm{~cm}^{-1}$ and $3000-2800 \mathrm{~cm}^{-1}$ are due to $\mathrm{OH}$ and $\mathrm{CH}$ stretching, respectively. The observed frequencies in the $1500-1200 \mathrm{~cm}^{-1}$ range are assigned to the coupled

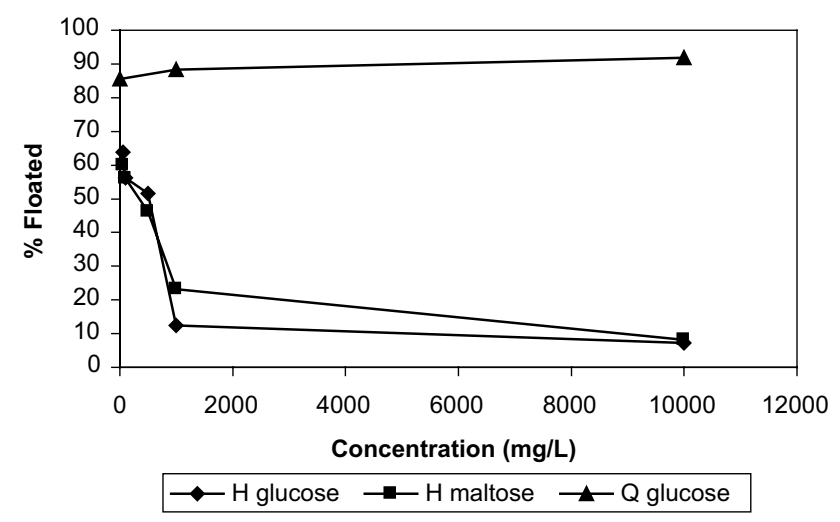

Fig. 4. Floatability of hematite $(\mathrm{H})$ and quartz $(\mathrm{Q})$ as a function of the monomer and dimer depressant concentrations. 


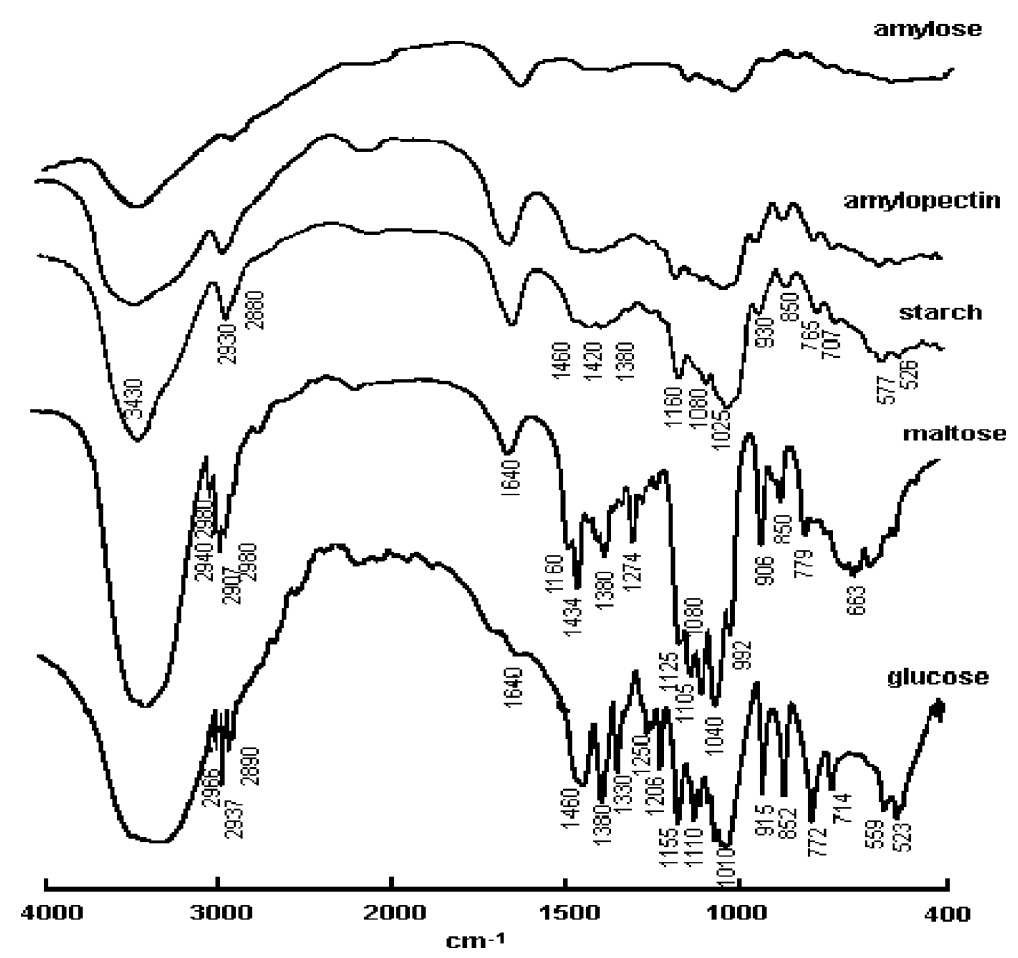

Fig. 5. Infrared transmission spectra $\left(\mathrm{KBr}, \mathrm{cm}^{-1}\right)$ of amylose, amylopectin, starch, maltose and glucose.

modes of vibration that involve atomic groups with hydrogen atoms. In the $1200-1000 \mathrm{~cm}^{-1}$ region, a group of intense bands are observed. The region between 1000 and $700 \mathrm{~cm}^{-1}$ shows bands due to the atomic group involved in the anomeric form (Dauchez et al., 1992; Cael et al., 1975). It is difficult to eliminate all water in the samples, thus bands due to absorptions of water molecules were observed. The bands located at about $1460,1280,1250,990$ and $850 \mathrm{~cm}^{-1}$ are assigned to the vibrations associated with the $\mathrm{CH}_{2}$ group. The frequencies near 1340, 1060 and $1040 \mathrm{~cm}^{-1}$ were demonstrated to be due to modes involving deformations of $\mathrm{C}-\mathrm{OH}$ groups. The modes related with $\mathrm{CCH}$ bending were identified at about 1420, 1206 and 1070, while the $\mathrm{C}-\mathrm{O}$ and the $\mathrm{C}-\mathrm{C}$ stretching contribute to modes related to the bands at 1155,1110 and $915 \mathrm{~cm}^{-1}$ (Sekkal et al., 1995).

The spectra of the carbohydrates adsorbed onto hematite are shown in Fig. 6. These are difference spectra corresponding to the adsorption tests for glucose (a), amylose (b), amylopectin (c) and starch (d) onto hematite obtained by spectrum subtraction technique. Only the $1500-750 \mathrm{~cm}^{-1}$ range is showed because this range was considered the real relevant one. The spectra are very similar indicating that the observed frequencies arise from similar vibrational modes. The most intense bands for the adsorbed carbohydrate spectra occurred at approximately 1150,1050 and $1020 \mathrm{~cm}^{-1}$. The band at $1150 \mathrm{~cm}^{-1}$, due to $\mathrm{C}-\mathrm{O}-\mathrm{C}$ vibrations, was observed only in the adsorbed polymer spectra. The major dif-

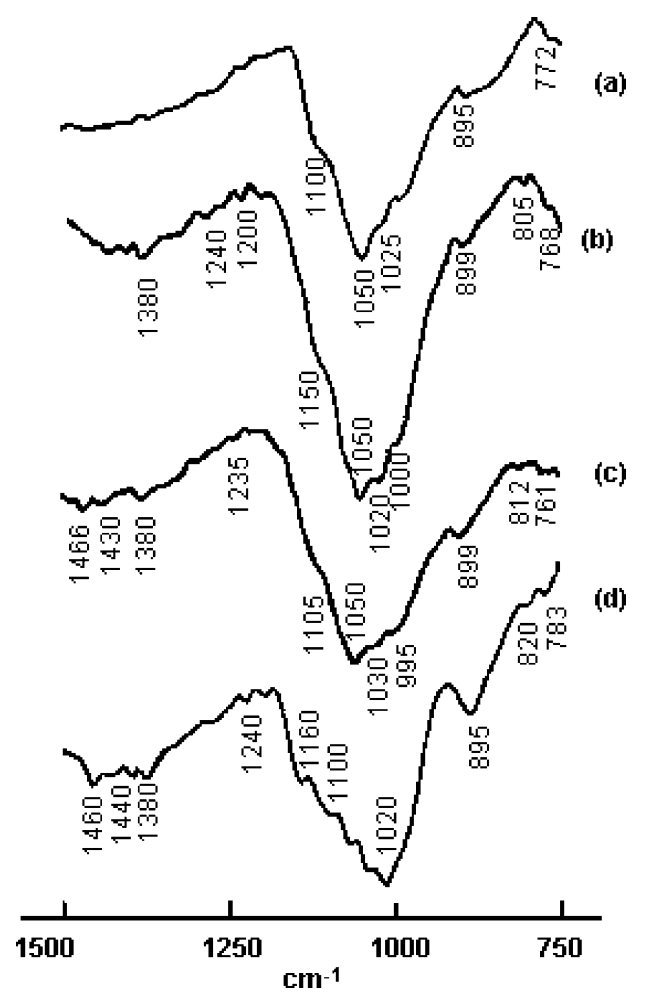

Fig. 6. Infrared transmission difference spectra $\left(\mathrm{KBr}, \mathrm{cm}^{-1}\right)$ of hematite with glucose (a), amylose (b), amylopectin (c) and starch (d) adsorbed.

ferences between the non-adsorbed and the adsorbed carbohydrates occurred in the $1000-700 \mathrm{~cm}^{-1}$ range, as 


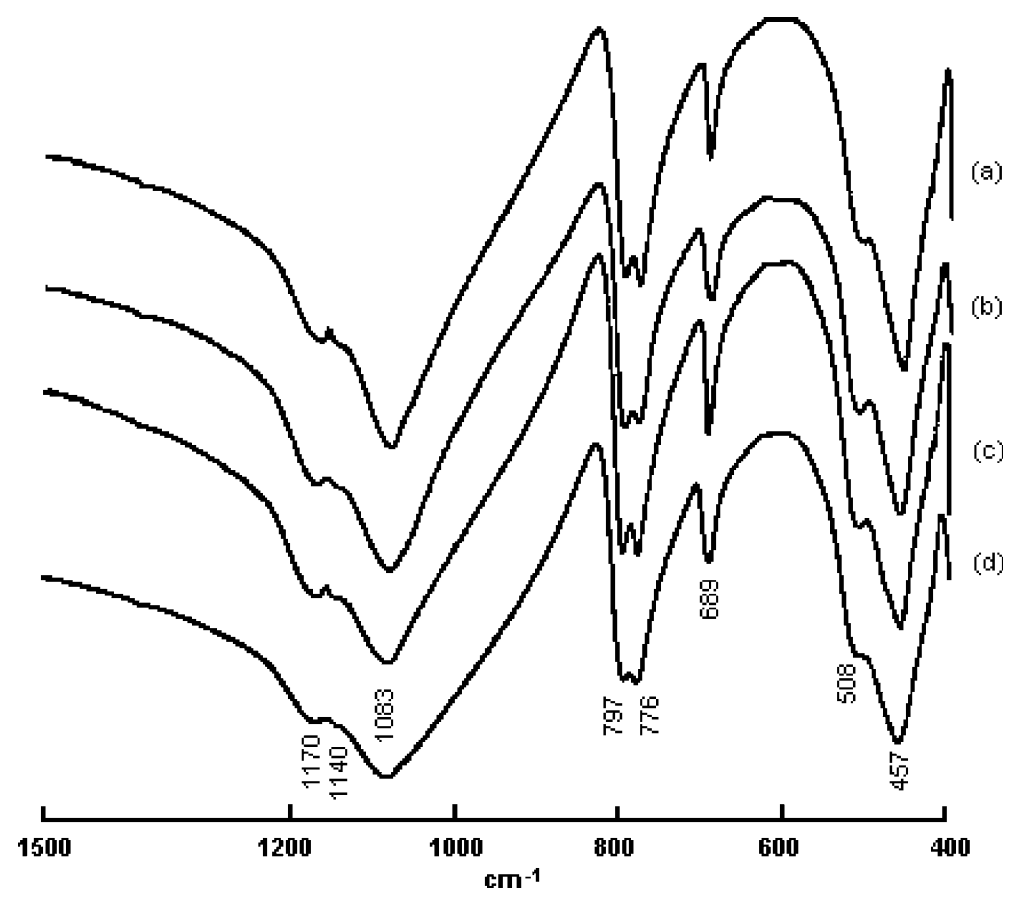

Fig. 7. Infrared transmission spectra $\left(\mathrm{KBr}, \mathrm{cm}^{-1}\right)$ of quartz after contact with: starch (a), amylose (b), amylopectin (c) and glucose (d).

also observed by Weissenborn et al. (1995) and Lima and Brandao (1999). The band at $915 \mathrm{~cm}^{-1}$, assigned to the asymmetric stretching ring vibration, was not detected in any adsorbed carbohydrate spectra. On the other hand, a band at about $895 \mathrm{~cm}^{-1}$ appeared in all spectra of the adsorbed carbohydrate. This band may correspond to the one originally at $850 \mathrm{~cm}^{-1}$, now shifted, assigned to the equatorial $\mathrm{C} 1 \mathrm{H}$ and $\mathrm{CH}_{2}$ deformation, or it may be really a new band. Another band at about $810 \mathrm{~cm}^{-1}$ was detected only in the adsorbed polymer spectra, which was also observed in other reported spectra (Weissenborn et al., 1995; Lima and Brandao, 1999).

The spectra of the non-adsorbed glucose (monomer) and maltose (dimer) were different as compared to the polymers' (Fig. 5); however, after adsorption, all the spectra became almost identical (Fig. 6). This demonstrated that the small molecules have adsorbed following the same interactions as the very large polymer molecules.

The adsorption of carbohydrates on quartz was not observed, by FTIR spectrometry as shown in Fig. 7. There are two possible reasons. Quartz has very intense IR absorption bands in the same spectra region where carbohydrates important bands occur, what makes spectral subtraction not useful. The relatively small amount of starch adsorbed onto quartz is not sufficient to show absorption bands on IR spectra, i.e. it was below the detection limit for the analysis, under the conditions attempted. This aspect was made worse by the relatively low specific surface area value of the quartz sample $\left(1.0 \mathrm{~m}^{2} / \mathrm{g}\right)$.

\section{Conclusions}

All the carbohydrates tested have been effective in maintaining hematite hydrophilic, including glucose and maltose; as for quartz only a flocculation action (mainly by amylopectin) had a mild effect in decreasing its floatability by amine collector.

It was not possible to correlate the depressant action and the amount of polysaccharide adsorbed, mainly for quartz. Depression is a complex phenomenon, in which adsorption is only one step, although being the most important one.

Glucose was strongly bonded to the hematite surface (adsorbed); even after thoroughly washing the hematite particles with water, it was not possible to remove it away from the surface, as proved by the infrared technique. The interaction mechanism between the hematite surface and the monomer/dimer was the same as the one occurring with the polymers, since the non-adsorbed spectra, which were originally different, have become almost identical after adsorption.

On the basis of the IR spectral information it is reasonable to conclude that a chemical complex (chemisorption) is formed between starch and the iron atoms on the hematite surface: a new band was observed at about $810 \mathrm{~cm}^{-1}$, which was not assigned for the original hematite or carbohydrate. The alternative hypothesisreaction of starch hydroxyl groups with hematite surface hydroxyls - would cause the disappearance or change in frequency from the spectra of bands due to vibrations of specific hydroxyl groups, what was not observed. 
The depressant action of the carbohydrates on hematite could occur without any flocculation action (as evidenced in the glucose microflotation tests), although this aggregation probably is positive to flotation, mainly in industrial conditions; on the contrary, for quartz depression a flocculation effect is necessary.

\section{References}

Cael, J.J., Koenig, J.L., Blackwell, J., 1975. Infrared and Raman spectroscopy of carbohydrates. Part VI: Normal coordinate analysis of V-amylose. Biopolymers 14, 1885-1903.

Dauchez, M., Derreumax, P., Vergoten, G., 1992. Vibrational molecular force field of model compounds with biologic interest. II. Harmonic dynamics of both anomers of glucose in the crystalline state. Journal of Computational Chemistry 14, 263-277.

Dubois, M.K.A., Gilles, J.K., Hamilton, P.A.R., Smith, F., 1956. Calorimetric method for determination of sugars and related substances. Analytical Chemistry 28, 350-356.

Hogg, R., 1999. Polymer adsorption and flocculation. In: Laskowski, J.S. (Ed.), Polymers in Minerals Processing. Montreal, pp. 3-17.
Lima, R.M.F., Brandao, P.R.G., 1999. Investigation on the selectivity in the inverse flotation of iron ores by infrared spectrometry. In: Laskowski, J.S. (Ed.), Polymers in Minerals Processing. Montreal, pp. 139-152.

Liu, Q., Zhang, Y., Laskowski, J.S., 2000. The adsorption of polysaccharides onto mineral surfaces: an acid/base interaction. International Journal of Mineral Processing 60, 229-245.

Peres, A.E.C., Correa, M.I., 1996. Depression of iron oxides with corn. Minerals Engineering 9, 1227-1234.

Schulz, N.F., Cooke, S.R.B., 1953. Froth flotation of iron ores: adsorption of starch products and laurylamine acetate. Industrial and Engineering Chemistry 45, 2767-2772.

Sekkal, M., Dincq, V., Legrand, P., Huvenne, J.P., 1995. Investigation of the linkages in several oligosaccharides using FT-IR and FT Raman spectroscopies. Journal of Molecular Structure 349, 349352.

Weissenborn, P.K., Warren, L.J., Dunn, J.G., 1995. Selective flocculation of ultrafine iron ore. 1. Mechanism of adsorption of starch onto hematite. Colloids and Surfaces A 99, 11-27.

Weissenborn, P.K., Warren, L.J., Dunn, J.G., 1996. Behaviour of amylopectin and amylose components of starch in the selective flocculation of ultrafine iron ore. International Journal Mineral Processing 47, 197-211. 